\title{
Solar-cell efficiency enhancements using 2D photonic crystals
}

\section{Pablo Aitor Postigo}

Increased performance can also be achieved for photon-sensing devices such as photodetectors and thermo-photovoltaic devices.

The performance of solar cells (SCs) can be enhanced significantly by employing 2D photonic crystals (2D-PCs). They can either be used as omnidirectional, broadband antireflection coatings (ARCs) or applied to thin-film SCs (where PC effects are much more significant than in generic SCs). Although multilayer ARCs are commonly used, problems related to material selection, thermal mismatch, sensitivity to thickness variations, and instability of the thin-film stacks persist. Tapered, submicrometer patterned structures may offer a more practical method for ultrabroadband, omnidirectional, antireflection operation. ${ }^{1}$ We have developed an optimized PC ARC that combines good optical properties (low reflectivity over a wide range of wavelengths) with high mechanical stability based on application of a thin dielectric layer perforated with the PC pattern. (A slight patterning of the $\mathrm{PC}$ inside the indium phosphide- $\mathrm{InP}-$ layer is required for optimum performance.) Thus, mixed, thin dielectric-layer/bulk patterning can be used for best optical and mechanical results compared to alternatives such as motheye-like fabrication, nanorods, and nanostructures employing oblique-angle deposition methods, which may be damaged seriously even by smooth contact with other surfaces.

Our results show that our fabricated nanoholes/PC structures can be used as effective ARCs. Changing the lattice symmetry (square and triangular) does not introduce drastic changes in reflectivity, although the reflectivity is reduced for smaller lattice parameters $(a)$. Figure 1 shows the reflectivity measured at normal incidence for our two best-performing coatings, one corresponding to a triangular and the other to a square lattice with $a=470$ and 400nm, respectively. For comparison, we also show the reflectivity of a nonpatterned InP bulk sample. The values obtained are comparable to those characterizing other tapered,

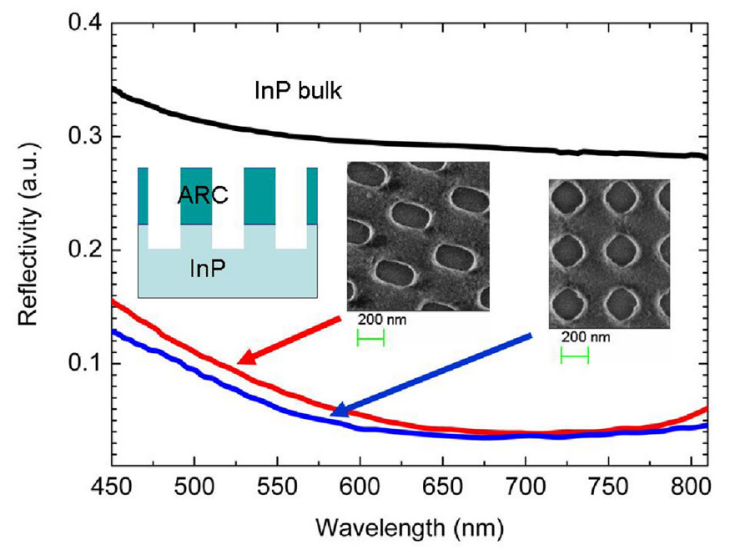

Figure 1. Reflectivity (in arbitrary units, a.u.) measured at normal incidence for our best-performing coatings, corresponding to triangular and square lattices with lattice parameters $a=470$ and $400 \mathrm{~nm}$, respectively. For comparison, the corresponding reflectivity of an indium phosphide (InP) bulk sample is also included. ARC: Antireflection coating.
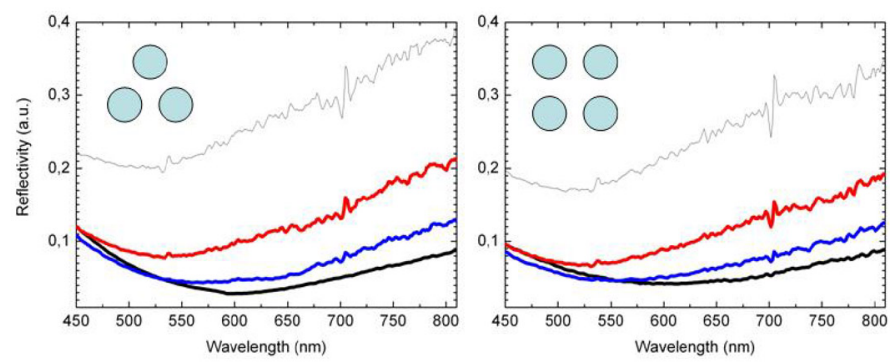

Figure 2. Reflectivity of the photonic-crystal (PC) ARC shown in Figure 1 for four angles of incidence (from top to bottom: 75, 65, 55, and $\left.45^{\circ}\right)$. Lower reflectivities correspond to smaller angles of incidence. The angle is formed between the normal to the surface and the incident light.

Continued on next page 

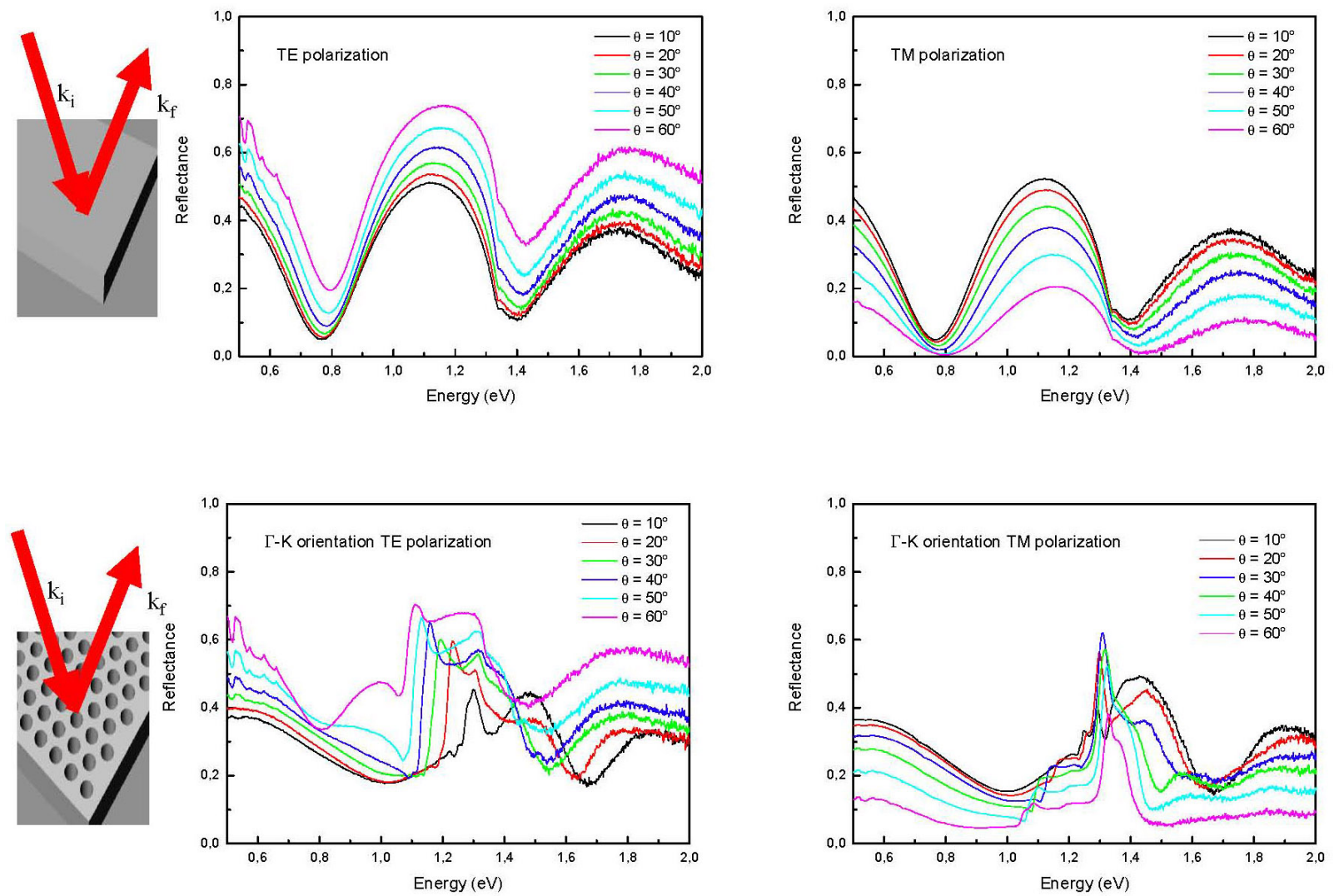

Figure 3. Reflectance of our InP nanopatterned sample for transverse electric (TE) and transverse magnetic (TM) polarization along the $\Gamma-K$ direction of the reciprocal lattice and (top) for the same layer without any patterning. $k_{i}, k_{f}$ : Moments of the incident and reflected light. $\theta$ : Angle of incidence.

subwavelength-etched structures, although the mechanical resistance of our nanopatterned film could be better because of our use of holes instead of columns. In both samples, the total etching depths were 140nm through the ARC and 160nm inside the InP layer. Deeper etching can greatly enhance the antireflection effect, since the refractive index changes smoothly and continuously, but further work is needed to constrain the optimum value.

The influence of the incident angle of light on SC efficiency is crucial for device performance. Figure 2 shows the reflectivity of our PC ARC for four angles of incidence. In general, the efficiency of SCs with a flat surface decreases rapidly with increasing incident angle because of increased reflection losses. These can decrease SC efficiency by more than $50 \%$ at angles of incidence of $\sim 70^{\circ}$. In the PC ARC, however, the reflectivity is sustained at incident angles $>60^{\circ}$, while it is degraded by only $20 \%$ at $75^{\circ}$. This angle-independent performance is required for the device to operate efficiently during all daylight hours.
The effects of nanopatterned PC structures can be better recognized with decreasing thickness of the layer in which they are made. This may be of particular relevance for thin-layer SCs, for which the well-known effects of PC slabs can be used to enhance the performance of the final device. In general, in addition to diffraction, resonant effects on the absorption can be expected because of the presence of photonic modes that can be 'felt' to some extent, depending on the depth of the etched holes and the slab thickness. ${ }^{2,3}$ We have measured the reflectivity and transmission of thin (270nm-thick) InP layers that can be of interest to get an initial idea of the expected real magnitude of the effect. Our samples were thin layers of InP nanopatterned with holes down to the glass substrate in a triangular lattice with $a=450 \mathrm{~nm}$ and $r / a=0.32$ (where $r$ is the radius of the nanoholes). The transmission and reflectance were measured by angle-resolved reflectivity from 0.55 to $2.0 \mathrm{eV}$ (600 to $2250 \mathrm{~nm}$ ). Figure 3 shows the nanopatterned sample's reflectance for the 

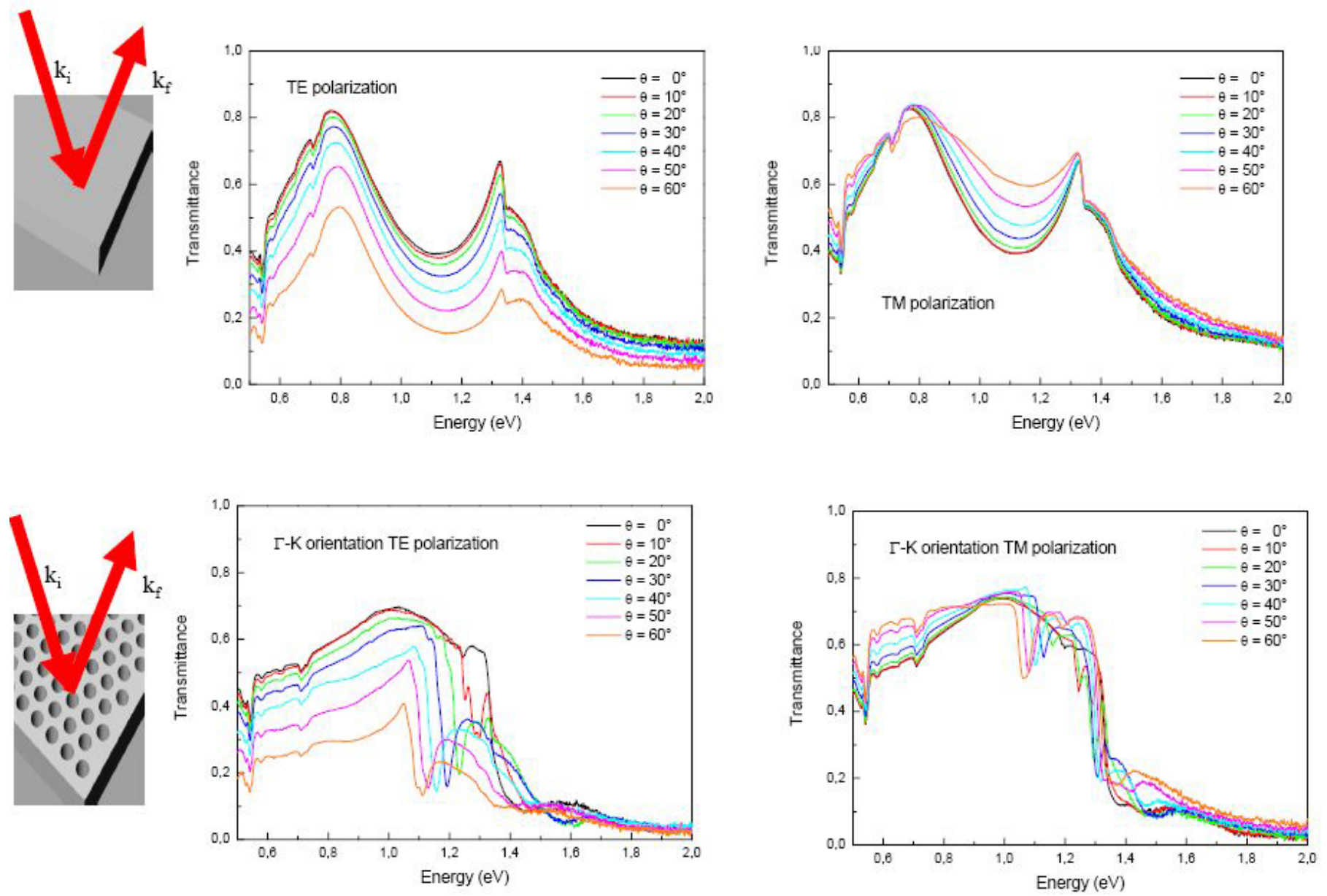

Figure 4. Transmittance of the InP PC sample of Figure 3 for TE and TM polarization along the $\Gamma-K$ direction of the reciprocal lattice. (top) Without patterning.

transverse electric (TE) and transverse magnetic (TM) polarizations and for different angles of incidence along the $\Gamma-K$ direction of the reciprocal lattice, as well as for the same layer without any patterning.

In general, there are noticeable differences between the spectra of the PC and nonpatterned samples. The minima and maxima of the oscillations are shifted with respect to the nonpatterned sample, while new features have appeared that significantly alter the overall line shape. In particular, the sharper features from 0.8 to $1.8 \mathrm{eV}$ (1550nm to $688 \mathrm{~nm}$ ) increase or decrease the total reflectance of the PC sample. They correspond to the well-known Fano-like resonances of the discrete photonic modes of the PC lattice. They have been used to experimentally determine the position of the PC bands. ${ }^{4}$ Although absorption by the InP layer should be very low in the range of wavelengths measured, we designed our experiment to also measure its transmittance (see
Figure 4). The spectra of both samples again show clear differences.

Manufacturing and characterizing a complete SC with a 2DPC front surface involving subwavelength motifs may be of interest. Even with partial vertical confinement (caused by, for example, a nonfavorable distribution of the index of refraction), all reasons discussed so far justify the use of a PC front surface with subwavelength motifs. We have manufactured a singlejunction germanium/indium gallium phosphide SC with a PC nanostructured surface. ${ }^{5}$ We achieved an increase in photocurrent of up to $8 \%$ using a SC with $40 \%$ of its surface covered by a PC pattern. We measured enhancements of the external quantum efficiency by $22 \%$ for a wide range of wavelengths, and up to a $46 \%$ for specific wavelengths, which clearly correlates with 
the PC-patterned area. Diffraction caused by the PC ARC was the main cause of the efficiency enhancement, but we still need to separate it from other photonic effects.

In summary, we have validated our approach to enhance the performance of SCs using PCs, which could also be applied to other photon-sensing devices, including photodetectors and thermo-photovoltaic devices. Further work is needed to understand in depth all possible photonic effects involved, from classical diffraction towards the inside of the semiconductor material to subwavelength diffraction, variation of the index of refraction due to the photonic bands, change of the lifetime of the photons by the PC layer, or role of the submicronic roughness produced during etching and fabrication of the PC SC. Moreover, special efforts are required to derive optimized PC geometries and designs (such as thickness) for these devices. We are now applying these results to manufacture 2D-PC-enhanced solar cells.

The author acknowledges project grants Consolider GENESIS CSD2006-0004 (Spanish Ministry of Education and Culture) and NANOGEFFES ENE2009-14481-C02-02.

\section{Author Information}

\section{Pablo Aitor Postigo}

Institute of Microelectronics of Madrid

Tres Cantos, Spain

Pablo Aitor Postigo is interested in design, fabrication, and characterization of PCs for new optoelectronic devices, including PC lasers, solar cells, and quantum photonic devices.

\section{References}

1. J. Y. Chen and K. W. Sun, Enhancement of the light conversion efficiency of silicon solar cells by using nanoimprint anti-reflection layer, Sol. Energy Mater. Sol. Cells 94, pp. 629-633, 2010 .

2. Y. Park, E. Drouard, O. El Daif, X. Letartre, P. Viktorovitch, A. Fave, A. Kaminski, M. Lemiti, and C. Seassal, Absorption enhancement using photonic crystals for silicon thin film solar cells, Opt. Express 17 (16), pp. 14312-14321, 2009.

3. S. Zanotto, M. Liscidini, and L. C. Andreani, Light trapping regimes in thin-film silicon solar cells with a photonic pattern, Opt. Express 18 (5), pp. 4260-4274, 2010.

4. V. N. Astratov, D. M. Whittaker, I. S. Culshaw, R. M. Stevenson, M. S. Skolnick,

T. F. Krauss, and R. M. De La Rue, Photonic band-structure effects in the reflectivity of periodically patterned waveguides, Phys. Rev. B 60, p. R16255, 1999.

5. I. Prieto, B. Galiana, P. A. Postigo, C. Algora, L. J. Martínez, and I. Rey-Stolle, Enhanced quantum efficiency of Ge solar cells by a two-dimensional photonic crystal nanostructured surface, Appl. Phys. Lett. 94 (19), pp. 191102-191105, 2009. 